NTP RESEARCH REPORT ON

SYNTHETIC TURF/RECYCLED TIRE CRUMB RUBBER: FEASIBILITY

STUDY IN SUPPORT OF NON-INHALATION IN ViVO

EXPOSURES OF SYNTHETIC TURF/ ReCyCled Tire Crumb RubBer

NTP RR 13

JULY 2019 


\section{NTP Research Report on Synthetic Turf/Recycled Tire Crumb Rubber: Feasibility Study in Support of Non-inhalation In Vivo Exposures of Synthetic Turf/Recycled Tire Crumb Rubber}

Research Report 13

National Toxicology Program

July 2019

National Toxicology Program

Public Health Service

U.S. Department of Health and Human Services

ISSN: 2473-4756

Research Triangle Park, North Carolina, USA 
Synthetic Turf/Recycled Tire Crumb Rubber: Feasibility Study in Support of Non-inhalation In Vivo Exposures of Synthetic Turf/Recycled Tire Crumb Rubber

\section{Table of Contents}

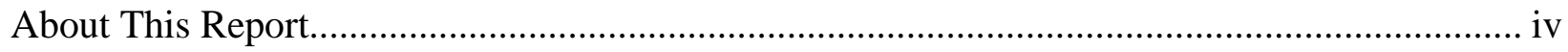

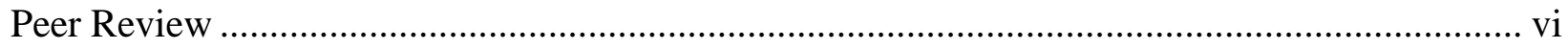

Publication Details ........................................................................................................... vii

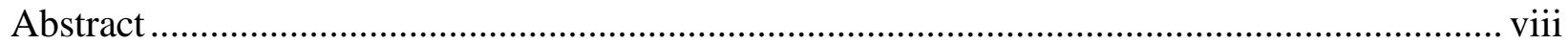

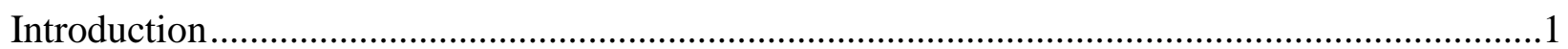

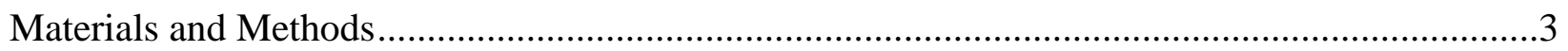

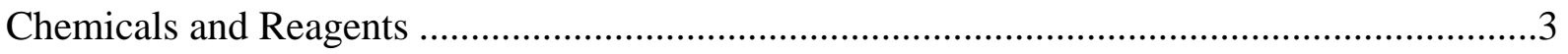

Preparation of Crumb Rubber Test Material....................................................................

Sieving of Crumb Rubber Test Material....................................................................

Feasibility Testing of Potential Crumb Rubber Formulations ...............................................4

Preparation of Dermal Formulations ....................................................................

Preparation of Oral Gavage Formulations ...............................................................

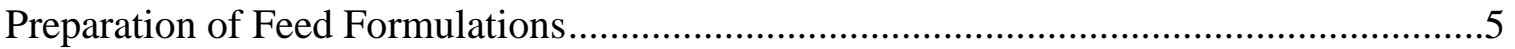

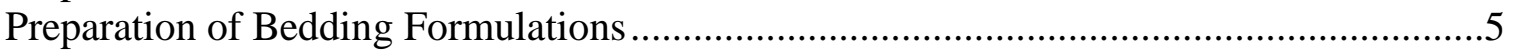

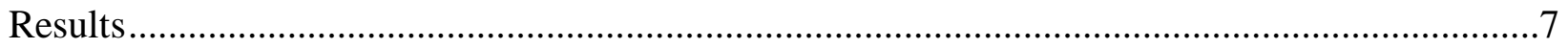

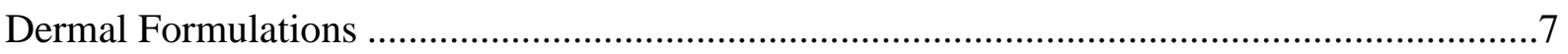

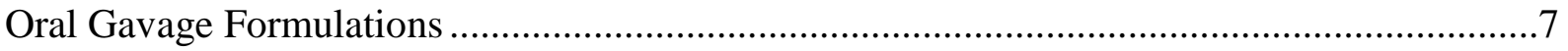

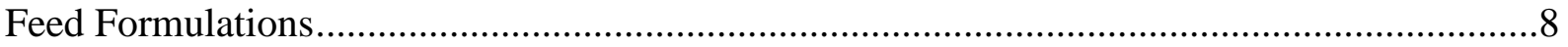

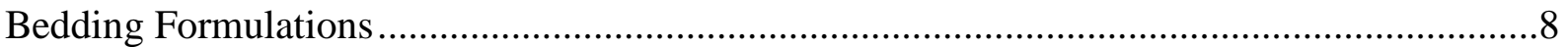

Additional Bedding Feasibility Testing Following Preliminary Testing ...............................8

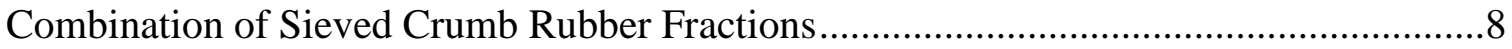

Bedding Formulation Using Combined Fraction .......................................................

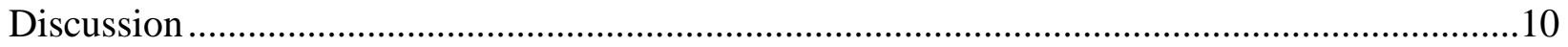

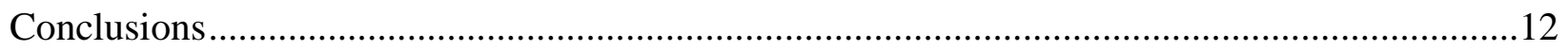

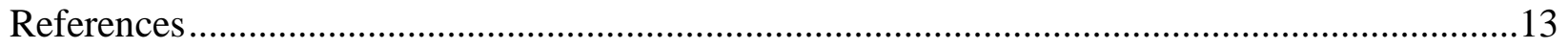

\section{Tables}

Table 1. Crumb Rubber Particle Size Ranges Following Original Sieving...............................15

Table 2. Crumb Rubber Feasibility Testing Design ............................................................15

Table 3. Crumb Rubber Particle Size Ranges Following Feasibility Testing Completion ...........15 


\section{Figures}

Figure 1. Digital Image of Crumb Rubber Bulk Material .........................................................16

Figure 2. Digital Image of Crumb Rubber at 80-Mesh Particle Size Showing Nylon Bundles.

Figure 3. Representative Digital Images of 80-Mesh Crumb Rubber in Ethanol Showing Clumping of Material During Dispensing

Figure 4. Representative Digital Images of 400-Mesh Crumb Rubber in Ethanol Showing Clumping of Material During Dispensing.

Figure 5. Representative Digital Images of 80-Mesh Crumb Rubber in 0.5\% Aqueous Methylcellulose Showing Clumping of Material During Dispensing.

Figure 6. Representative Digital Images of 400-Mesh Crumb Rubber in Corn Oil Demonstrating Dispensing from Plastic Needle (Left) and Homogeneity (Right)

Figure 7. Representative Digital Images of Homogeneity of 14-Mesh Crumb Rubber in Feed Formulation after Shaking on Days 0 (A), 1 (B), 4 (C), and 7 (D) .

Figure 8. Representative Digital Images of the Homogeneity of the 40-Mesh Crumb Rubber in Feed Formulation after Shaking on Days 0 (A), 1 (B), 4 (C), and 7 (D)

Figure 9. Representative Digital Images of Homogeneity of 14-Mesh (Left) and 40-Mesh (Right) Crumb Rubber in Bedding Formulation after Shaking on Days 0 (Top), 1 (Middle), and 4 (Bottom)

Figure 10. Representative Digital Images of Homogeneity of Combined Crumb Rubber in Bedding Formulation Before Shaking (Left) and After Shaking (Right)

Figure 11. Digital Image of Combined Crumb Rubber Fraction

Figure 12. Digital Image of the 400-Mesh Crumb Rubber Fraction 
Synthetic Turf/Recycled Tire Crumb Rubber: Feasibility Study in Support of Non-inhalation In Vivo Exposures of Synthetic Turf/Recycled Tire Crumb Rubber

\section{About This Report}

National Toxicology Program ${ }^{1}$

${ }^{1}$ Division of the National Toxicology Program, National Institute of Environmental Health Sciences, Research Triangle Park, North Carolina, USA

\section{Collaborators}

Jamie S. Richey, Heather M. Toy, Karen E. Elsass, Dawn M. Fallacara, Georgia K. Roberts, Matthew D. Stout, Barney R. Sparrow

Battelle, Columbus, Ohio, USA

Jamie S. Richey, M.S.

Heather M. Toy, B.S.

Karen E. Elsass, B.S.

Dawn M. Fallacara, Ph.D.

Barney R. Sparrow, Ph.D.

Division of the National Toxicology Program, National Institute of Environmental Health Sciences, Research Triangle Park, North Carolina, USA

Georgia K. Roberts, Ph.D.*

Matthew D. Stout, Ph.D.*

*To whom the correspondence should be addressed.

\section{Contributors}

Division of the National Toxicology Program, National Institute of Environmental Health Sciences, Research Triangle Park, North Carolina, USA

Provided overall programmatic guidance and review

Abee L. Boyles, Ph.D.

John R. Bucher, Ph.D.

Michael J. DeVito, Ph.D.

Darlene Dixon, Ph.D.

Michelle J. Hooth, Ph.D.

Dave E. Malarkey, Ph.D., D.V.M.

Cynthia V. Rider, Ph.D. 
Provided internal peer review

Anika L. Dzierlenga, Ph.D.

Suzanne E. Fenton, Ph.D.

Esra Mutlu, Ph.D.

Provided oversight of external peer review

Mary S. Wolfe, Ph.D.

\section{ICF, Durham, North Carolina, USA}

Prepared reports and conducted peer review

David F. Burch, M.E.M., Principal Investigator

Susan E. Blaine, B.A.

Natalie K. Blanton, M.P.H.

Jeremy S. Frye, M.S.L.S

Lindsey M. Green, M.P.H.

Tara Hamilton, M.S.

Katherine R. Helmick, M.P.H.

Penelope E. Kellar, M.S.

Whitney K. Mitchell, B.S. 
Synthetic Turf/Recycled Tire Crumb Rubber: Feasibility Study in Support of Non-inhalation In Vivo Exposures of Synthetic Turf/Recycled Tire Crumb Rubber

\section{Peer Review}

The draft research report, NTP Research Report on Synthetic Turf/Recycled Tire Crumb Rubber: Feasibility Study in Support of Non-inhalation In Vivo Exposures of Synthetic Turf/Recycled Tire Crumb Rubber, was evaluated by the reviewers listed below. These reviewers served as independent scientists, not as representatives of any institution, company, or governmental agency. In this capacity, reviewers determined if the design and conditions of these NTP studies were appropriate and ensured that this NTP Research Report presented the experimental results and conclusions fully and clearly.

\section{Peer Reviewers}

\section{William J. Brock, Ph.D., DABT, Fellow ATS}

Owner

Brock Scientific Consulting, LLC Montgomery Village, Maryland, USA

\section{Kristini K. Miles, Ph.D., DABT}

President

Venture Chemical Consulting, LLC

Atlanta, Georgia, USA 
Synthetic Turf/Recycled Tire Crumb Rubber: Feasibility Study in Support of Non-inhalation In Vivo Exposures of Synthetic Turf/Recycled Tire Crumb Rubber

\section{Publication Details}

Publisher: National Toxicology Program

Publishing Location: Research Triangle Park, North Carolina

ISSN: $2473-4756$

DOI: https://doi.org/10.22427/NTP-RR-13

Report Series: NTP Research Report Series

Report Series Number: 13

Official citation: National Toxicology Program (NTP). 2019. NTP research report on synthetic turf/recycled tire crumb rubber: feasibility study in support of non-inhalation in vivo exposures of synthetic turf/recycled tire crumb rubber. Research Triangle Park, NC: National Toxicology Program. Research Report 13. 
Synthetic Turf/Recycled Tire Crumb Rubber: Feasibility Study in Support of Non-inhalation In Vivo Exposures of Synthetic Turf/Recycled Tire Crumb Rubber

\section{Abstract}

Public health concern for playing on synthetic turf fields with crumb rubber infill has increased in recent years. Crumb rubber manufactured from recycled automobile tires contains potential carcinogenic and toxic substances, and, with over 12,000 synthetic turf fields in the United States, the potential for exposure is widespread. The National Toxicology Program (NTP) conducted research to improve the understanding of potential human exposure to crumb rubber and its biological activity. The objective of the studies as described in this report was to evaluate likely exposure scenarios in humans that could be translated into exposure routes for in vivo testing. Feasibility trials, without animals, were conducted to evaluate various crumb rubber formulations for bedding (incidental contact), feed (indirect ingestion), oral gavage (direct consumption), and dermal (direct contact) exposures.

Due to the physical characteristics of crumb rubber (irregularly sized particles of ground tires encompassing a large range in size and composition), particles were physically manipulated prior to testing. Milling was not feasible due to the characteristics of rubber (elasticity and thermal properties) and the additives employed during the grinding process; therefore, crumb rubber was sieved using standard mesh sizes into various particle sizes for evaluation in each exposure scenario. Formulations for testing of the various exposure scenarios were prepared at concentrations that allowed for maximum exposure to the crumb rubber material. Bedding and feed formulations, using sieved fraction sizes of crumb rubber greater than $420 \mu \mathrm{m}$, were prepared at 50:50 (wt:wt) crumb rubber:bedding and at 50,000 ppm crumb rubber in feed, respectively. Feed formulations were rotated on an orbital shaker at ambient temperature for 7 days to evaluate uniformity. Bedding formulations were rotated on an orbital shaker at ambient or elevated temperature for 4 days to evaluate uniformity and potential for vapor off-gassing. Uniformity of the feed formulations was maintained over 7 days of shaking. Larger crumb rubber particles (approximately 1,410 $\mu \mathrm{m}$ or greater) maintained uniformity with the bedding, but smaller particles (less than $1,410 \mu \mathrm{m}$ ) settled to the bottom of the cage. No vapor off-gassing was observed for the bedding.

Corn oil gavage formulations were prepared as homogenous suspensions at concentrations up to $200 \mathrm{mg} / \mathrm{mL}$ using crumb rubber with a particle size no greater than $170 \mu \mathrm{m}$. Particle sizes and concentrations greater than $170 \mu \mathrm{m}$ resulted in blockage of the gavage needle during dispensing. Dermal administration of crumb rubber suspensions of any size fraction tested was determined not to be feasible due to clumping of crumb rubber in the vehicle, preventing homogeneous formulations. From this study, three potential human exposure scenarios (incidental exposure, indirect ingestion, and direct consumption) were identified as feasible exposure regimens for in vivo testing. 
Synthetic Turf/Recycled Tire Crumb Rubber: Feasibility Study in Support of Non-inhalation In Vivo Exposures of Synthetic Turf/Recycled Tire Crumb Rubber

\section{Introduction}

Synthetic turf fields are widely used in the United States, and their use is expanding. Although such fields historically have been installed in professional sports complexes, they are becoming increasingly popular in community recreational areas, including schools and public parks. These expanded applications include areas across the United States that are used by people of all ages, including young children. In a synthetic turf field, infill materials are spread between the "grass" fibers to provide cushioning and traction. Today, most commonly, the infill consists of granulated rubber pellets referred to as crumb rubber. Crumb rubber is manufactured by shredding used or recycled automobile tires. Recycled tires contain numerous, potentially carcinogenic and toxic substances, either as components of the original tire rubber or accumulated during normal use. While information is known about tire manufacturing, many variables are unknown, including persistence of byproducts, chemical changes during vulcanization, and adsorption of environmental chemicals, which might affect the chemical composition of the ultimate crumb rubber product. A recent literature review of crumb rubber chemical characterization studies outlines some chemicals of concern; using ADMET Predictor $^{\mathrm{TM}}$, this study identified 197 predicted carcinogens, of which 52 have been previously classified as carcinogens by the U.S. Environmental Protection Agency (EPA) or European Chemicals Agency (ECHA) ${ }^{1}$. Other chemicals of concern as well as more information about crumb rubber manufacturing and synthetic turf construction and standards, are described in the 2016 EPA Federal Research Action Plan².

Public concerns about potential health impacts associated with the use of synthetic turf fields have risen dramatically in recent years due to the chemical composition of crumb rubber and the opportunity for widespread, frequent, and long-term exposure, particularly for young athletes. Considering that information to evaluate whether there are adverse health effects from playing on synthetic turf fields is currently limited, several governmental organizations at the international, federal, and state level recently launched research efforts to better understand human exposure and human health hazards focusing on crumb rubber. Internationally, $\mathrm{ECHA}^{3}$ and the National Institute for Public Health and the Environment (RIVM) ${ }^{4}$ of the Netherlands have evaluated the health risks of playing sports on synthetic turf with crumb rubber. The Federal Research Action Plan ${ }^{5-7}$, which involves several U.S. federal agencies, aims to determine and fill important knowledge gaps (FRAP 2016, Appendix B), characterize constituents of recycled tire crumb, and identify ways that people are exposed to tire crumb rubber through typical field use activities. The California Office of Environmental Health Hazard Assessment $(\mathrm{OEHHA})^{8}$ is evaluating exposure scenarios, characterizing new and in-field crumb rubber, and developing biomonitoring protocols.

In 2015, OEHHA nominated synthetic turf/crumb rubber to the National Toxicology Program (NTP) for short-term in vivo and in vitro studies to generate information and improve understanding of potential health impacts of chemicals released from synthetic turf, with an emphasis on crumb rubber. The NTP research program ${ }^{9}$ developed in response to this request used in vitro and in vivo systems to address uncertainties about potential human exposure to hazardous components of crumb rubber. The NTP research approach included both in vivo and in vitro studies and considered the most likely routes of human contact with crumb rubber (e.g., ingestion, dermal contact, and inhalation). The study objective was to investigate which exposure 
conditions could have biological effects, with a focus on characterizing the bioaccessibility and systemic exposure of crumb rubber constituents.

The results of the NTP studies on crumb rubber are communicated in a series of Research Reports ${ }^{10-13}$. The crumb rubber used in the NTP studies was provided by OEHHA specifically for research purposes, and consisted of fresh recycled tire crumb rubber obtained from manufacturing facilities. The physical and chemical characteristics of the material were evaluated using a variety of analytical methods ${ }^{10}$. The characterization work provided an understanding of the material used in the NTP studies and helped interpret chemical analyses in the in vivo and in vitro studies. Characterization of the NTP material will allow interpretation of NTP study findings in relation to crumb rubber evaluated as part of the Federal Research Action Plan (FRAP) and OEHHA research efforts, including samples from new and weathered material, and samples from indoor and outdoor fields. For in vitro testing, multiple cell lines (representing skin, lung, and small intestine) were used to evaluate the extractability of crumb rubber and to look for evidence of biological activity of crumb rubber constituents via measurements of cytotoxicity ${ }^{11}$. For in vivo studies, feasibility testing was conducted to determine what routes of exposure were possible ${ }^{12}$. On the basis of this testing, 14-day in vivo studies were performed ${ }^{13}$ using oral exposure and exposure in bedding. Dermal studies were not conducted on the basis of feasibility work ${ }^{12}$, and feasibility testing for particle and vapor inhalation studies is ongoing.

The purpose of the work presented in this publication was to determine what conditions would be feasible for conducting an in vivo study with crumb rubber, with the focus on oral and dermal routes of exposure. This report characterizes the challenges associated with dose administration and exposure of crumb rubber that likely would be encountered while performing in vivo toxicology studies via probable non-inhalation routes of human exposure. It also describes the testing performed to determine and support the feasibility of conducting bedding, feed, oral gavage, and dermal exposures in vivo. 
Synthetic Turf/Recycled Tire Crumb Rubber: Feasibility Study in Support of Non-inhalation In Vivo Exposures of Synthetic Turf/Recycled Tire Crumb Rubber

\section{Materials and Methods}

\section{Chemicals and Reagents}

OEHHA provided the crumb rubber used in these studies. The material, manufactured by either an ambient or a cryogenic manufacturing process, is fresh crumb rubber. Three lots were received in multiple 1-L glass jars. First, the material from one facility and one type of processing was combined to produce three lots, each approximately $5 \mathrm{~kg}$. Subsequently, all material was combined into one lot (Lot No. CRM06092016). Physical and chemical characterization of this lot is described elsewhere ${ }^{10}$. The crumb rubber was stored at approximately $5^{\circ} \mathrm{C}$.

The following materials were obtained and used with no further purification: ethanol (95\%, USP grade) from Sigma Chemical (St. Louis, MO); corn oil (nonfood grade) from Spectrum Chemical (New Brunswick, NJ); and methylcellulose (USP grade, 4,000 cps) from Spectrum Chemical (New Brunswick, NJ). Hardwood bedding (Sanichips) from P.J. Murphy Forest Products Corporation (Mt. Jewett, PA). Rodent urine (Sprague Dawley Rat) and feces (Sprague Dawley Rat) from Bioreclamation (Baltimore, MD). NTP-2000 feed from Ziegler Bros., Inc. (Gardners, PA).

\section{Preparation of Crumb Rubber Test Material}

Crumb rubber is a heterogeneous composite of ground automobile tires and, as a final product, has a wide particle size distribution ${ }^{14}$. Automobile tires are manufactured from vulcanized rubber and various reinforcing materials. Vulcanization is used to cure and strengthen the materials and can impart organosulfur compounds (from catalysts), zinc oxide, and stearic acid (for crosslinking and curing) to the product. Metal (steel) reinforcements are commonly added. Common rubber matrices include synthetic styrene-butadiene or blends of natural rubber and synthetic styrene-butadiene. Other additives, such as petroleum oils or various specialty chemicals, can be added to improve flexibility or reduce friction. Tire recycling into crumb rubber also can include environmental components such as sand, calcite, asphalt, road dust, and tar. The lot of crumb rubber used for this testing was characterized as reported by $\mathrm{NTP}^{10}$.

Particle sizes for ground crumb rubber used as turf filler range from 0.6 to $2.5 \mathrm{~mm}^{15}$. Mechanically ground tires typically have higher inhalable fractions than cryogenically ground rubber tires, although the latter process can be controlled to produce smaller-sized particles for uses in other applications. Based on the particle size distribution determined for this lot of material as shown in Figure 1 and as reported in $\mathrm{NTP}^{10}$, the need for reduction of the particle size was identified in order to accommodate size limitations/restrictions of various methods of dose administration (such as gavage). Due to the physical characteristics of crumb rubber (irregular particles of ground tires that encompass a large size range and composition), physical manipulation methods were performed prior to feasibility testing. Milling was not feasible due the elasticity and thermal properties of rubber and the additions of talc or quartz during the grinding process. Therefore, the crumb rubber was sieved into various particle size ranges for evaluation in each exposure scenario. 
Synthetic Turf/Recycled Tire Crumb Rubber: Feasibility Study in Support of Non-inhalation In Vivo Exposures of Synthetic Turf/Recycled Tire Crumb Rubber

\section{Sieving of Crumb Rubber Test Material}

The crumb rubber was sieved to generate material of different particle sizes and to determine the amount of material available in the different particle size ranges for testing the various routes of administration. The crumb rubber fractions were isolated from the bulk material by sieving using U.S. Standard Brass Test Sieves (Fisher) stacked on a WS Tyler® RO-TAP® (Mentor, OH) sieve shaker. Table 1 summarizes the fractions produced, the amount isolated, and the intended use. During sieving, the remainder of the nylon cord fibers that comprise the tires agglomerated and formed bundles in the material, as shown in Figure 2. Any visible bundles and fibers were manually removed from the material using forceps prior to use. The crumb rubber fractions were stored at approximately $5^{\circ} \mathrm{C}$.

\section{Feasibility Testing of Potential Crumb Rubber Formulations}

During the design phase, limitations related to the physical nature of the crumb rubber test article and formulation specifications for each exposure scenario were evaluated. Crumb rubber particles (which are irregularly sized particles of ground tires encompassing a large particle size range and composition) were physically manipulated prior to testing to determine the particle size distribution of the bulk material. To maximize the potential for successful evaluation of multiple routes of exposure, there was a desire to explore ways of reducing the crumb rubber particle size. Milling the crumb rubber material was deemed not feasible due to the characteristics of rubber (elasticity and thermal properties) and the additives that would have been required to be employed during the grinding process (such as quartz and talc).

As an alternative to milling, the crumb rubber was sieved to separate the material into particle sizes fractions, allowing the use of the smaller particle sizes for certain routes of exposure. Table 1 summarizes the fractions produced, the quantity of each fraction isolated, and the intended use. The quantity of each particle size fraction available following sieving was taken into consideration for design of the study. Formulations for testing for the various exposure scenarios were prepared at concentrations that allowed for maximum exposure to the crumb rubber material. The final outline of the feasibility testing performed to support dermal and oral routes of administration for in vivo toxicity studies were performed as summarized in Table 2.

\section{Preparation of Dermal Formulations}

For dermal studies, the use of a solvent interface was desired to produce uniform application of the crumb rubber to the skin. Suspensions of crumb rubber in ethanol were prepared at a target concentration of $300 \mathrm{mg} / \mathrm{mL}$ using each of the 80-mesh (particle size range, 170-420 $\mu \mathrm{m}$ ) and the 400-mesh (particle size range, $37-170 \mu \mathrm{m}$ ) materials. The suspensions were mixed vigorously for approximately 15 minutes using a stir bar and stir plate, then again mixed for 5 minutes using a Silverson (East Longmeadow, MA) mixer.

\section{Preparation of Oral Gavage Formulations}

For gavage studies, an 18- to 22-gauge gavage needle is used to administer formulations to rodents. The needle size limitation $(20$-gauge $=$ approximately $600 \mu \mathrm{m}$ inner diameter) eliminated the 14- and 40-mesh particle size fractions for consideration of use in a gavage study. Suspensions of crumb rubber in corn oil and $0.5 \%$ aqueous methylcellulose were prepared at a 
Synthetic Turf/Recycled Tire Crumb Rubber: Feasibility Study in Support of Non-inhalation In Vivo Exposures of Synthetic Turf/Recycled Tire Crumb Rubber

target concentration of $200 \mathrm{mg} / \mathrm{mL}$ using the 80-mesh (particle size range 170-420 $\mu \mathrm{m}$ ) material and the 400-mesh (particle size range $37-170 \mu \mathrm{m}$ ) material.

\section{Preparation of Feed Formulations}

Formulations of crumb rubber mixed with irradiated NTP-2000 meal feed were prepared at a target concentration of 50,000 ppm to evaluate homogeneity and stability. A 50,000-ppm concentration was selected as the maximum concentration for a feed toxicity study where the rodents might consume the crumb rubber. Formulations were prepared using either 14-mesh (particle size range greater than 1,410 $\mu \mathrm{m}$ ) or 40-mesh (particle size range 420-1,410 $\mu \mathrm{m}$ ) material. The potential for rodents to consume the feed while ignoring the crumb rubber pellets of these mesh sizes was discussed, however due to the limited quantity of the smaller size fraction available for testing, the 14- and 40-mesh sizes were evaluated for feed formulation.

The formulations were prepared by manually combining and stirring feed (approximately $57 \mathrm{~g}$ ) and crumb rubber (approximately $3 \mathrm{~g}$ ) in individual $60-\mathrm{mL}$ widemouthed glass bottles. Bulk preparation of feed formulations was not possible due to the potential settling of the crumb rubber during repeated handling to fill feeders. To simulate animal room samples, crumb rubber:feed mixtures containing 5\% urine and feces were also prepared. The presence of urine and feces in a formulation can potentially cause the crumb rubber particles to clump together and inadvertently cause inhomogeneity over time. Testing in the presence of urine and feces allowed for the confirmation of homogeneity under simulated conditions of the animals being present.

Each bottle containing either formulation or formulation with urine and feces was vigorously shaken on an orbital shaker at ambient temperature for approximately 60 minutes. After shaking, the sample container was visually examined to assess the homogeneity of the mixture. Following feed preparation and testing, the remaining crumb rubber formulations were stored open to air at ambient temperature to simulate use conditions in the animal rooms and the testing repeated on Days 1,4 , and 7.

\section{Preparation of Bedding Formulations}

For bedding studies, the use of crumb rubber in place of hardwood bedding in the animal cages was discussed with the laboratory veterinary and animal care staff. Due to the nonabsorptive nature of crumb rubber, maintaining a dry living environment for the animals was problematic. Based on animal welfare concerns, using crumb rubber alone as bedding material was excluded as a testing condition. Instead, a combination of crumb rubber and hardwood bedding was used. The maximum ratio of crumb rubber:bedding mixture selected that would maintain a dry living environment in the animal cages was determined to be 50:50 (wt:wt).

Formulations of crumb rubber mixed with bedding material were prepared in a 50:50 (wt:wt) crumb rubber:bedding mixture for evaluation of homogeneity and stability. Formulations were prepared using either the 14-mesh (particle size range greater than 1,410 $\mu \mathrm{m}$ ) or the 40-mesh (particle size range $420-1,410 \mu \mathrm{m}$ ) material. The formulations were prepared by manually combining and stirring equal amounts of each material into individual animal cages. Bulk preparation of bedding formulations was not possible due to the potential settling of the crumb rubber during repeated handling to add bedding to cages. A bedding-only control and bedding:crumb rubber mixtures containing 5\% urine and feces to simulate animal room samples were also prepared. The presence of urine and feces in a formulation can potentially cause the 
crumb rubber particles to clump together and inadvertently cause inhomogeneity over time. Testing in the presence of urine and feces allowed for the confirmation of homogeneity under simulated conditions of the animals being present.

Each animal cage containing bedding, formulation, or formulation with urine and feces was shaken vigorously on an orbital shaker at ambient temperature for approximately 30 minutes. A volatile organic carbon monitor (MiniRae 3000, RAE Systems, San Jose, CA) was used to measure the presence of any vapors containing volatile organic carbon in the air above the bedding at time points of 5, 10, 15, and 30 minutes. After shaking, each animal cage was visually inspected to assess the overall homogeneity of the mixture. In addition, the process was repeated at elevated temperatures for approximately 15 minutes. A heat lamp was placed above the mixture while the animal cage and constituents were shaken on an orbital shaker. Temperature was monitored while the material was rotated. Following bedding preparation and testing, the remaining crumb rubber formulations were stored open to air at ambient temperature to simulate use conditions in the animal rooms and the testing repeated on Days 1 and 4. 
Synthetic Turf/Recycled Tire Crumb Rubber: Feasibility Study in Support of Non-inhalation In Vivo Exposures of Synthetic Turf/Recycled Tire Crumb Rubber

\section{Results}

\section{Dermal Formulations}

For both the 80 - and 400-mesh crumb rubber, the resulting $300 \mathrm{mg} / \mathrm{mL}$ mixtures were not homogeneous; the mixtures consisted of clumped crumb rubber that could not be drawn into pipettes or syringes for dispersal (Figure 3 and Figure 4). The volatility of ethanol as a solvent, and other solvents suitable for dermal studies (such as acetone and water mixtures), created enhanced surface interactions that caused the crumb rubber to agglomerate, precluding preparation of homogenous suspensions for dermal applications. The preferred vehicles for dermal studies are $95 \%$ ethanol or acetone due to volatilization following application without causing significant skin irritation on repeated use. Incremental dilution of the formulations with solvent down to final concentrations of approximately $215 \mathrm{mg} / \mathrm{mL}$ did not improve the homogeneity. Using the procedures described and based on the testing results, preparation of formulations of crumb rubber in the solvents necessary to perform a dermal study were deemed infeasible.

\section{Oral Gavage Formulations}

The 80-mesh (particle size range 170-420 $\mu \mathrm{m}$ ) material was initially used to assess gavageability and potential clumping with $0.5 \%$ aqueous methylcellulose. The same clumping of crumb rubber observed in the ethanol suspensions for dermal formulations was observed for the methylcellulose gavage suspension (Figure 5). Gavageability of the 80-mesh methylcellulose formulation was not feasible due to the clumping and a crumb rubber particle size that was too large for the 20-gauge gavage needle.

A suspension of crumb rubber in corn oil was prepared using the 400-mesh (particle size range 37-170 $\mu \mathrm{m}$ ) material. The corn oil formulation suspension was homogenous and could be drawn into a syringe without the gavage needle attached (Figure 6) but not with the needle attached. Aliquots $(1 \mathrm{~mL})$ of the formulation could be dispensed repeatedly utilizing the same syringe within approximately 3 seconds using a metal 20-gauge gavage needle attached and within approximately 7 seconds using a plastic 20 -gauge gavage needle attached.

Following gavageability testing, the remaining crumb rubber formulation in corn oil was stored at approximately $5^{\circ} \mathrm{C}$ for 7 days. On Day 7 , the formulation was allowed to come to ambient temperature and then was mixed for approximately 15 minutes with a stir bar and stir plate to resuspend the mixture. The resulting suspension was homogeneous, although appeared thicker in consistency compared to that on Day 0. Due to the thicker consistency of the formulation, gavageability of the formulation was reevaluated. Aliquots $(1 \mathrm{~mL})$ of the formulation could be dispensed repeatedly using the same syringe within approximately 7 seconds using a metal 20gauge gavage needle attached rather than within 3 seconds, as observed on Day 0. Based on the testing results, formulations of crumb rubber using the 400-mesh (particle-size range 37$170 \mu \mathrm{m}$ ) material in corn oil could be prepared as homogenous suspensions at concentrations up to $200 \mathrm{mg} / \mathrm{mL}$ and were deemed acceptable for use in animals based on the specification requiring gavage delivery within 4 seconds for $1 \mathrm{~mL}$. While the deliver time at Day 7 exceeded 4 seconds, the volume for delivery required for a mouse study would be approximately $0.3 \mathrm{~mL}$ (based on body weight) versus the $1 \mathrm{~mL}$ used in these feasibility studies. Certain processes are 
needed at the time of dose administration during an in vivo study, such as pulling up the formulation into the syringe without the gavage needle in place and wiping down the outside of the syringe prior to needle attachment.

\section{Feed Formulations}

No visible differences in homogeneity were observed for any formulation mixture prepared at $50,000 \mathrm{ppm}$, regardless of the particle size of crumb rubber used and whether urine and feces were present (Figure 7 and Figure 8). Based on the testing results, formulations of crumb rubber in NTP-2000 feed were deemed feasible for preparing homogenous formulations at concentrations up to $50,000 \mathrm{ppm}$ using any available particle size of the crumb rubber material when prepared as described.

\section{Bedding Formulations}

The crumb rubber formulations prepared with the 14-mesh (particle size range greater than $1,410 \mu \mathrm{m}$ ) material with or without the presence of urine and feces maintained their homogeneity throughout all stability testing days. The crumb rubber in the formulations prepared with the 40-mesh (particle size range 420-1,410 $\mu \mathrm{m}$ ) material rapidly settled to the bottom of the cage on Day 0 and remained settled throughout the four testing days regardless of whether urine and feces were present (Figure 9). A maximum temperature of $31^{\circ} \mathrm{C}$ was achieved during testing. No elevated volatile organic carbon levels were observed at any point throughout the stability determinations at either ambient or elevated temperature for any of the bedding formulations or the control bedding. Due to the observed settling, formulations of crumb rubber mixed with bedding material prepared in a 50:50 (wt:wt) crumb rubber:bedding mixture using the 40-mesh crumb rubber material, while feasible for use, are not recommended due to the inability to maintain a homogenous formulation. Formulations of crumb rubber mixed with bedding material prepared in a 50:50 (wt:wt) crumb rubber:bedding mixture using the 14-mesh (particle-size range greater than $1,410 \mu \mathrm{m}$ ) material, however, were deemed suitable for maintaining a homogenous formulation when prepared in individual animal cages.

\section{Additional Bedding Feasibility Testing Following Preliminary Testing}

\section{Combination of Sieved Crumb Rubber Fractions}

Following activities using the sieved crumb rubber fractions, the 40- and 80-mesh fractions were not specifically needed for the formulations for the individual routes of administration and thus were combined with the 14-mesh material. Recombining these size fractions maximized the material available for the studies. While the 40-mesh fraction did experience settling when mixed into bedding by itself, the impact this would have while using the recombined material was deemed negligible. Based on weight, the 40 -mesh particle size made up approximately $29 \%$ of the total recombined fraction and, even when settling occurs in bedding, this material would still be available to the animals, particularly when considering rodent nesting activities.

To recombine the fractions, the material was homogenized such that equal amounts of each fraction were combined in layers into two 20-L glass media bottles, with each fraction added in alternating layers. The alternating layers of each material encouraged distribution of the various 
particle sizes within the bottles. Headspace was maintained in the bottles to allow vigorous movement and mixing of the particles. The bottles were sealed, rotated on a jar roller, and mixed by inversion for a minimum of 10 minutes per round of mixing to induce appropriate movement and mixing of the material. Following the first round of mixing, the contents of each bottle were combined in alternating layers into additional $20-\mathrm{L}$ glass media bottles. The bottles were sealed, mixed on a jar roller, and inverted for a minimum of 10 minutes each. Three rounds of mixing of the test chemical were performed, resulting in a combined crumb rubber fraction $(14,40,80$ mesh) with a particle size range of greater than $170 \mu \mathrm{m}$.

The final crumb rubber fractions (combine greater than $170 \mu \mathrm{m}$ and 400-mesh with particle size range $37-170 \mu \mathrm{m})$ were stored at approximately $5^{\circ} \mathrm{C}$.

\section{Bedding Formulation Using Combined Fraction}

A formulation of the combined crumb rubber fraction (particle size range of greater than $170 \mu \mathrm{m}$ ) mixed with bedding material was prepared in a 50:50 (wt:wt) crumb rubber:bedding mixture for evaluation of homogeneity. Although some redistribution of the crumb rubber was observed, overall, the crumb rubber formulation prepared with the combined material maintained its homogeneity throughout testing (Figure 10). Based on the testing results, formulations of crumb rubber mixed with bedding material prepared in a 50:50 (wt:wt) crumb rubber:bedding mixture using the combined material material were deemed suitable for the preparation of homogenous formulations when formulated in individual animal cages. 
Synthetic Turf/Recycled Tire Crumb Rubber: Feasibility Study in Support of Non-inhalation In Vivo Exposures of Synthetic Turf/Recycled Tire Crumb Rubber

\section{Discussion}

Public health concern about exposure to crumb rubber while playing on synthetic turf fields with crumb rubber infill has increased in recent years. Crumb rubber manufactured from recycled tires contains potentially toxic and carcinogenic substances. Widespread exposure from the more than 12,000 synthetic turf fields in the United States is possible. NTP conducted research studies to improve the understanding of potential human exposure to crumb rubber and adverse health effects. The objective of these studies was to determine what routes of exposure would be feasible to conduct in vivo studies in mice as well as to communicate the challenges and considerations for designing an in vivo study with this unique material.

Methods to evaluate dermal exposure depend on time of exposure, surface contact efficiency, and surface loading. The attempt in this work to use $95 \%$ ethanol as a dispersion medium was based on the necessity for quick application on the test system as a monodisperse layer that mimicked skin exposure during sporting events. The preferred vehicles for dermal studies are 95\% ethanol or acetone due to volatilization following application without causing significant skin irritation on repeated use. Fast evaporation of the vehicle is preferred to avoid significant extraction of the crumb rubber. The application would provide a consistent, single layer of particles to avoid stacked material and impact on any transfer exposure efficiency. The observation that the crumb rubber particles were clumped in the ethanol solution indicates the material was not uniformly dispersed. The polarity of the solvent and subsequent poor wetting of the crumb rubber particles is a possible cause for increased particle interaction (note that when solutions were prepared in corn oil for gavageability, better dispersion was achieved). That the particle size distribution was not optimal for this application is also possible. Rodes et al. ${ }^{16}$, discussed efficiency of particle transfer for dermal application, and literature sources within that review mentioned the most frequently observed particle sizes transferring and remaining on skin were less than $100 \mu \mathrm{m}^{17}$. Although Kissel ${ }^{18}$ indicated wet particles larger than $150 \mu \mathrm{m}$ were transferred effectively, only $65-\mu \mathrm{m}$ particles were considered for dry transfer. Further, the particle size range used included 80-mesh $(170-420 \mu \mathrm{m})$ and 400-mesh $(37-170 \mu \mathrm{m})$ particles. This range is considerably larger than either size range mentioned in the Rodes review.

Similar particle size limitations are probable in the gavageability study for the oral gavage formulations. With corn oil as the dispersion solvent, the nonpolar solvent interactions between the rubber particle surfaces and the dispersant likely maintained wettable surfaces and added fluidity to the mixture. The added fluidity was most notable when using the 400-mesh particle size distribution, as the larger, 80-mesh particle size distribution did not sufficiently disperse in the corn oil matrix or the $0.5 \%$ aqueous methylcellulose matrix. The observation of poor uptake through the gavage needles (20 gauge $=$ approximately $600 \mu \mathrm{m}$ inner diameter) for the 400-mesh crumb rubber might be indicative of the nonuniform particle shapes of crumb rubber, their relative orientation to the needle orifice, and the impact of solvent wettability on surfaces (metal versus plastic). Further, the dispensing variance noted between metal and plastic needles suggests the needle materials affect dispensing differently. Differences in the dispensing time between freshly prepared and stored slurries suggests that clumping is not an isolated process (limited only to sufficiently large particle distribution, poor solvent choices, or both that result in poor wettability), but rather is a dynamic effect that reflects the heterogeneous composition of crumb rubber. 
Both the bedding and feed approaches demonstrate the impact of material size and flowability on solid material mixing and blending. Because particles must move relative to each other, their interactions are influenced by friction, interparticle forces, and gravity, with additional influences caused by mechanical interactions or liquid bridging effects. The difference between the 14-mesh and 40-mesh crumb rubber in bedding demonstrates the influence of friction and interparticle forces in which the small crumb rubber particle size distribution (40-mesh) is unable to maintain close association with the much larger bedding flakes and subsequently settle via gravity to the bottom. In contrast, the larger 14-mesh crumb rubber maintains a closer interaction with the bedding material with no substantial settling. While the 40-mesh fraction did experience settling when mixed into bedding, the impact for use of the recombined material was deemed negligible. Based on weight, the 40 -mesh particle size made up approximately $29 \%$ of the total recombined fraction. Though some settling of the material might occur, rodent burrowing and nesting behavior created an environment in which direct contact with the material would still occur. Interactions with feed exhibit similar influences, but the comparable sizes in the feed and crumb rubber particles resulted in less available air space. As such, both crumb rubber particle size distributions remained homogeneously mixed. 
Synthetic Turf/Recycled Tire Crumb Rubber: Feasibility Study in Support of Non-inhalation In Vivo Exposures of Synthetic Turf/Recycled Tire Crumb Rubber

\section{Conclusions}

The combined fraction (greater than 80 mesh, greater than $170 \mu \mathrm{m}$, Figure 11) and the 400-mesh fraction (Figure 12) were determined the acceptable fractions for use for in vivo toxicity studies (Table 3). The following assessments can be made based on the results of the feasibility work using the combined and 400-mesh test chemical fractions for potential crumb rubber toxicity studies:

1. Homogeneous dermal formulations containing the 80- and 400-mesh material cannot be prepared at concentrations up to $300 \mathrm{mg} / \mathrm{mL}$ using the procedures tested,

2. Gavage formulations can be prepared as homogenous suspensions in corn oil using only the 400-mesh material at concentrations up to $200 \mathrm{mg} / \mathrm{mL}$,

3. Feed formulations can be prepared as homogeneous formulations using material at any particle size at concentrations up to 50,000 ppm when formulated in individual glass jars and transferred to individual feeders, and

4. Bedding formulations can be prepared as homogeneous formulations using the combined material (greater than 80 mesh) in a 50:50 (wt:wt) crumb rubber:bedding mixture when formulated in individual animal cages.

Certain processes for gavage formulations in corn oil are needed at the time of dose administration during in vivo studies, such as drawing up the formulation into the syringe without the gavage needle in place and wiping down the outside of the syringe prior to needle attachment. The feed and bedding formulations must be prepared in individual glass jars and animal cages, respectively, at the time of use to prepare the appropriate concentration consistently and to preserve the homogeneity of the formulations. 
Synthetic Turf/Recycled Tire Crumb Rubber: Feasibility Study in Support of Non-inhalation In Vivo Exposures of Synthetic Turf/Recycled Tire Crumb Rubber

\section{References}

1. Perkins AN, Inayat-Hussain SH, Deziel NC, Johnson CH, Ferguson SS, Garcia-Milian R, Thompson DC, Vasiliou V. Evaluation of potential carcinogenicity of organic chemicals in synthetic turf crumb rubber. Environ Res. 2019; 169:163-172.

https://doi.org/10.1016/j.envres.2018.10.018

2. United States Environmental Protection Agency (U.S. EPA). Federal research action plan (FRAP) on recycled tire crumb used on playing fields and playgrounds. 2016. EPA/600/R16/364. https://www.epa.gov/sites/production/files/201612/documents/federal_research_action_plan_on_recycled_tire_crumb_used_on_playing_fields_a nd_playgrounds_status_report.pdf [Accessed: 3 Oct, 2017]

3. European Chemicals Agency (ECHA). Annex XV Report: An evaluation of the possible health risks of recycled rubber granules used as infill in synthetic turf sports fields. 2017. https://echa.europa.eu/documents/10162/13563/annexXV_report_rubber_granules_en.pdf/dbcb4ee6-1c65-af35-7a18-f6ac1ac29fe4

4. Rijksinstituut voor Volksgezondheid en Milieu [Netherlands National Institute for Public Health and the Environment] (RIVM). Evaluation of health risks of playing sports on synthetic turf pitches with rubber granulate. 2017. Report Number: 2017-0016. https://www.rivm.nl/dsresource?objectid=a397bdf3-7aa7-490a-85bd$\underline{5992 b 78 b c b 42 \& \text { type }=\text { pdf } \& \text { disposition }=\text { inline }}$

5. United States Environmental Protection Agency (U.S. EPA). Federal research on recycled tire crumb used on playing fields. U.S. Environmental Protection Agency; 2016.

https://www.epa.gov/chemical-research/federal-research-recycled-tire-crumb-used-playing-fields [Accessed: 2017]

6. Agency for Toxic Substances and Disease Registry (ATSDR). Federal research action plan on recycled tire crumb used on playing fields and playgrounds. 2016.

https://www.atsdr.cdc.gov/frap/index.html [Accessed: 2017]

7. Consumer Product Safety Commission (CPSC). Crumb rubber information center. 2016. https://www.cpsc.gov/Safety-Education/Safety-Education-Centers/Crumb-Rubber-SafetyInformation-Center [Accessed: 2017]

8. Office of Environmental Health Hazard Assessment (OEHHA). Synthetic turf studies. 2017. https://oehha.ca.gov/risk-assessment/synthetic-turf-studies [Accessed: 2017]

9. National Toxicology Program (NTP). Synthetic turf/recycled tire crumb rubber. 2017. https://ntp.niehs.nih.gov/results/areas/syntheticturf/index.html [Accessed: 2017]

10. National Toxicology Program (NTP). NTP research report on chemical and physical characterization of crumb rubber. Research Triangle Park, NC: National Toxicology Program; 2019. Research Report 11. https://doi.org/10.22427/NTP-RR-11

11. National Toxicology Program (NTP). NTP research report on synthetic turf/recycled tire crumb rubber: Characterization of the biological activity of crumb rubber in vitro. Research 
Synthetic Turf/Recycled Tire Crumb Rubber: Feasibility Study in Support of Non-inhalation In Vivo Exposures of Synthetic Turf/Recycled Tire Crumb Rubber

Triangle Park, NC: National Toxicology Program; 2019. Research Report 12. https://doi.org/10.22427/NTP-RR-12

12. National Toxicology Program (NTP). NTP research report on synthetic turf/recycled tire crumb rubber: Feasibility study in support of non-inhalation in vivo exposures of synthetic turf/recycled tire crumb rubber. Research Triangle Park, NC: National Toxicology Program; 2019. Research Report 13. https://doi.org/10.22427/NTP-RR-13

13. National Toxicology Program (NTP). NTP research report on synthetic turf/recycled tire crumb rubber: 14-day exposure characterization studies of crumb rubber in female mice housed on mixed bedding or dosed via feed or oral gavage. Research Triangle Park, NC: National Toxicology Program; 2019. Research Report 14. https://doi.org/10.22427/NTP-RR-14

14. Amari T, Themelis NJ, Wernick IK. Resource recovery from used rubber tires. Resources Policy. 1999; 25(3):179-188. https://doi.org/10.1016/S0301-4207(99)00025-2

15. Gomes J, Mota H, Bordado J, Cadete M, Sarmento G, Ribeiro A, Baiao M, Fernandes J, Pampulim V, Custodio M et al. Toxicological assessment of coated versus uncoated rubber granulates obtained from used tires for use in sport facilities. J Air Waste Manag Assoc. 2010; 60(6):741-746. https://doi.org/10.3155/1047-3289.60.6.741

16. Rodes CE, Newsome JR, Vanderpool RW, Antley JT, Lewis RG. Experimental methodologies and preliminary transfer factor data for estimation of dermal exposures to particles. J Expo Anal Environ Epidemiol. 2001; 11(2):123-139.

http://dx.doi.org/10.1038/sj.jea.7500150

17. Edwards RD, Lioy PJ. The EL sampler: A press sampler for the quantitative estimation of dermal exposure to pesticides in housedust. J Expo Anal Environ Epidemiol. 1999; 9(5):521529. http://dx.doi.org/10.1038/sj.jea.7500048

18. Kissel JC. Characterization of soil adherence to skin: Impact of historical misinterpretation of the Que Hee et al. data. Risk Anal. 1995; 15(6):613-614. http://dx.doi.org/10.1111/j.15396924.1995.tb01332.x 
Synthetic Turf/Recycled Tire Crumb Rubber: Feasibility Study in Support of Non-inhalation In Vivo Exposures of Synthetic Turf/Recycled Tire Crumb Rubber

Table 1. Crumb Rubber Particle Size Ranges Following Original Sieving

\begin{tabular}{cccc}
\hline Sieve Mesh & Particle Size Range $(\boldsymbol{\mu m})$ & Intended Use & Amount $(\mathbf{g})$ \\
\hline 14 & Greater than 1,410 & $\begin{array}{c}\text { Feed, bedding } \\
\text { (full particle size) }\end{array}$ & $6,999.01$ \\
40 & $420-1,410$ & $\begin{array}{c}\text { Feed, bedding } \\
\text { (reduced particle size) }\end{array}$ & $2,848.31$ \\
$80^{\mathrm{a}}$ & $170-420$ & Gavage, dermal & 38.71 \\
400 & $37-170$ & Gavage, dermal & 33.24 \\
Pan $^{\mathrm{b}}$ & $<37$ & NA & 0.27 \\
\hline
\end{tabular}

${ }^{\mathrm{a} C}$ Contained nylon fiber bundles along with particles.

${ }^{\mathrm{b} C}$ Contained only nylon fiber bundles.

Table 2. Crumb Rubber Feasibility Testing Design

\begin{tabular}{cccc}
\hline $\begin{array}{c}\text { Route of } \\
\text { Exposure }\end{array}$ & $\begin{array}{c}\text { Crumb Rubber } \\
\text { Mesh }\end{array}$ & Formulation Vehicle & Target Concentration \\
\hline Dermal & 80 & Ethanol & $300 \mathrm{mg} / \mathrm{mL}$ \\
& 400 & Ethanol & $300 \mathrm{mg} / \mathrm{mL}$ \\
Gavage & 80 & Corn oil or 0.5\% aqueous methylcellulose & $200 \mathrm{mg} / \mathrm{mL}$ \\
& 400 & Corn oil or 0.5\% aqueous methylcellulose & $200 \mathrm{mg} / \mathrm{mL}$ \\
Bedding & 14 & Bedding & $50: 50 \mathrm{w}: \mathrm{w}$ \\
& 40 & Bedding & $50: 50 \mathrm{w}: \mathrm{w}$ \\
Feed & 14 & Irradiated NTP-2000 feed & $50,000 \mathrm{ppm}$ \\
& 40 & Irradiated NTP-2000 feed & $50,000 \mathrm{ppm}$ \\
\hline
\end{tabular}

Table 3. Crumb Rubber Particle Size Ranges Following Feasibility Testing Completion

\begin{tabular}{cccc}
\hline Sieve Mesh & $\begin{array}{c}\text { Particle Size Range } \\
(\boldsymbol{\mu m})\end{array}$ & Intended Use & Amount(g) \\
\hline Combined & Greater than 170 & Feed, bedding & $8,327.37$ \\
(greater than 80 mesh) & & & \\
400 & $37-170$ & Gavage & 27.44 \\
Pan & $<37$ & NA & 0.27 \\
\hline
\end{tabular}


Synthetic Turf/Recycled Tire Crumb Rubber: Feasibility Study in Support of Non-inhalation In Vivo Exposures of Synthetic Turf/Recycled Tire Crumb Rubber

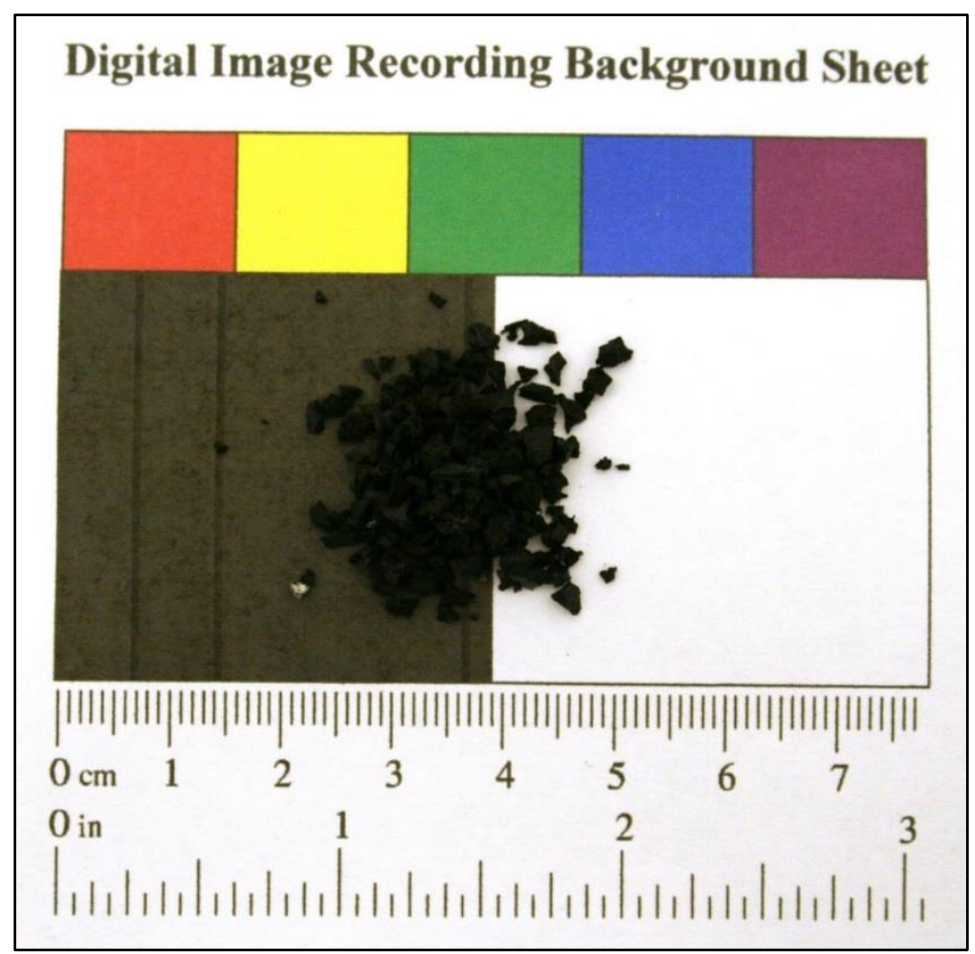

Figure 1. Digital Image of Crumb Rubber Bulk Material

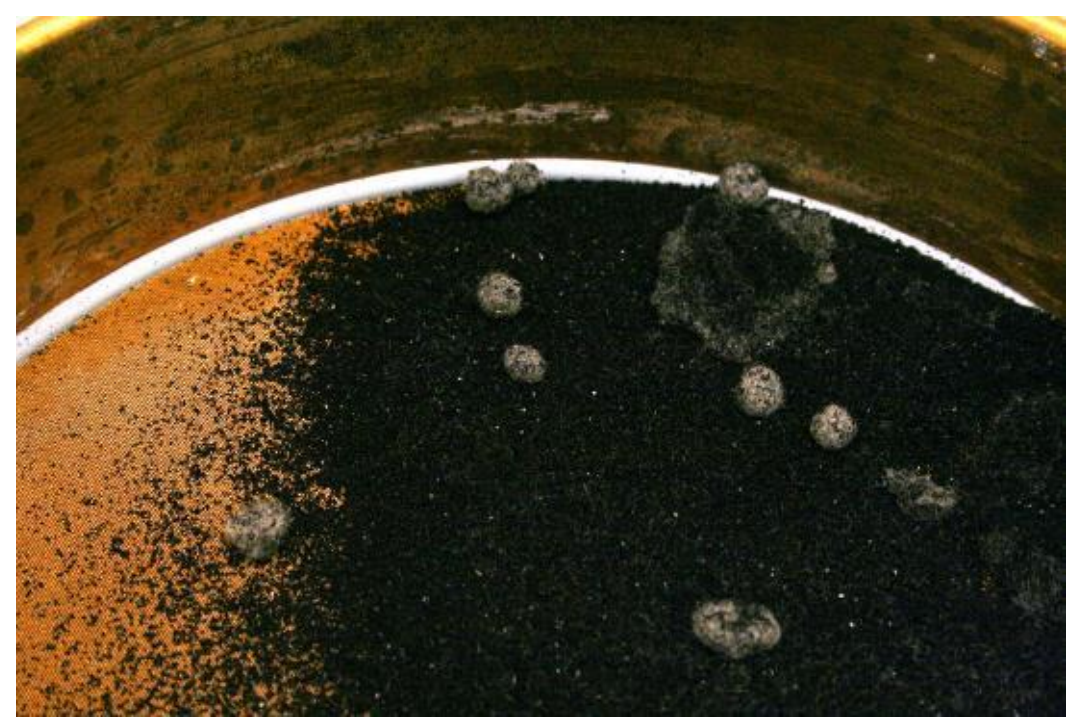

Figure 2. Digital Image of Crumb Rubber at 80-Mesh Particle Size Showing Nylon Bundles 
Synthetic Turf/Recycled Tire Crumb Rubber: Feasibility Study in Support of Non-inhalation In Vivo Exposures of Synthetic Turf/Recycled Tire Crumb Rubber
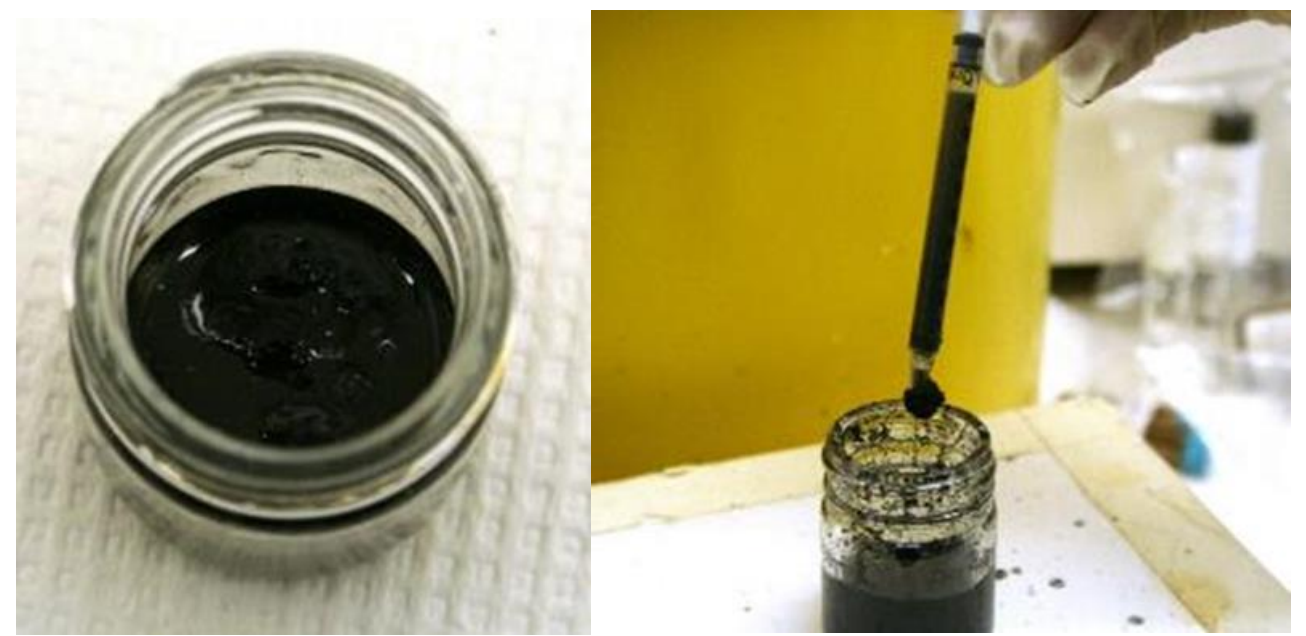

Figure 3. Representative Digital Images of 80-Mesh Crumb Rubber in Ethanol Showing Clumping of Material During Dispensing

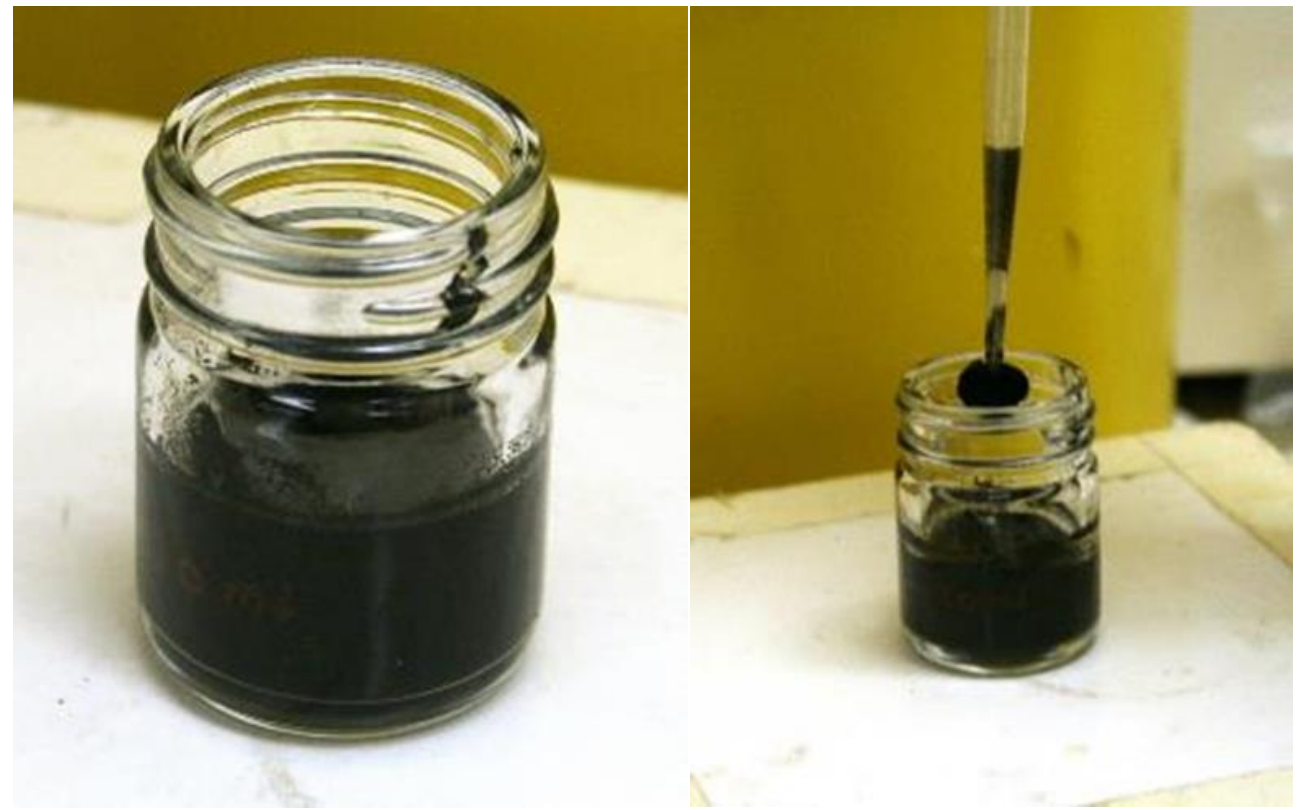

Figure 4. Representative Digital Images of 400-Mesh Crumb Rubber in Ethanol Showing Clumping of Material During Dispensing 
Synthetic Turf/Recycled Tire Crumb Rubber: Feasibility Study in Support of Non-inhalation In Vivo Exposures of Synthetic Turf/Recycled Tire Crumb Rubber

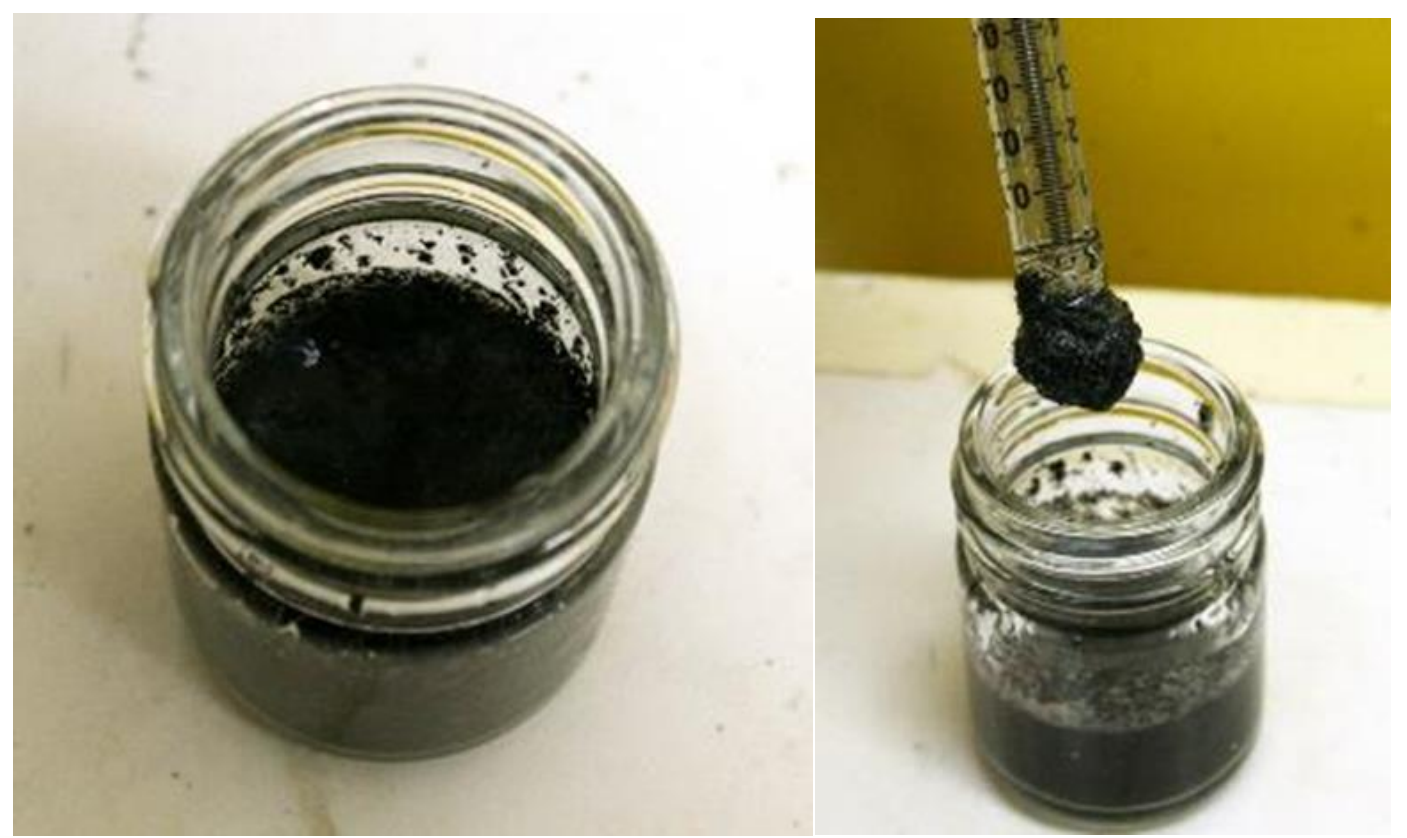

Figure 5. Representative Digital Images of 80-Mesh Crumb Rubber in 0.5\% Aqueous Methylcellulose Showing Clumping of Material During Dispensing
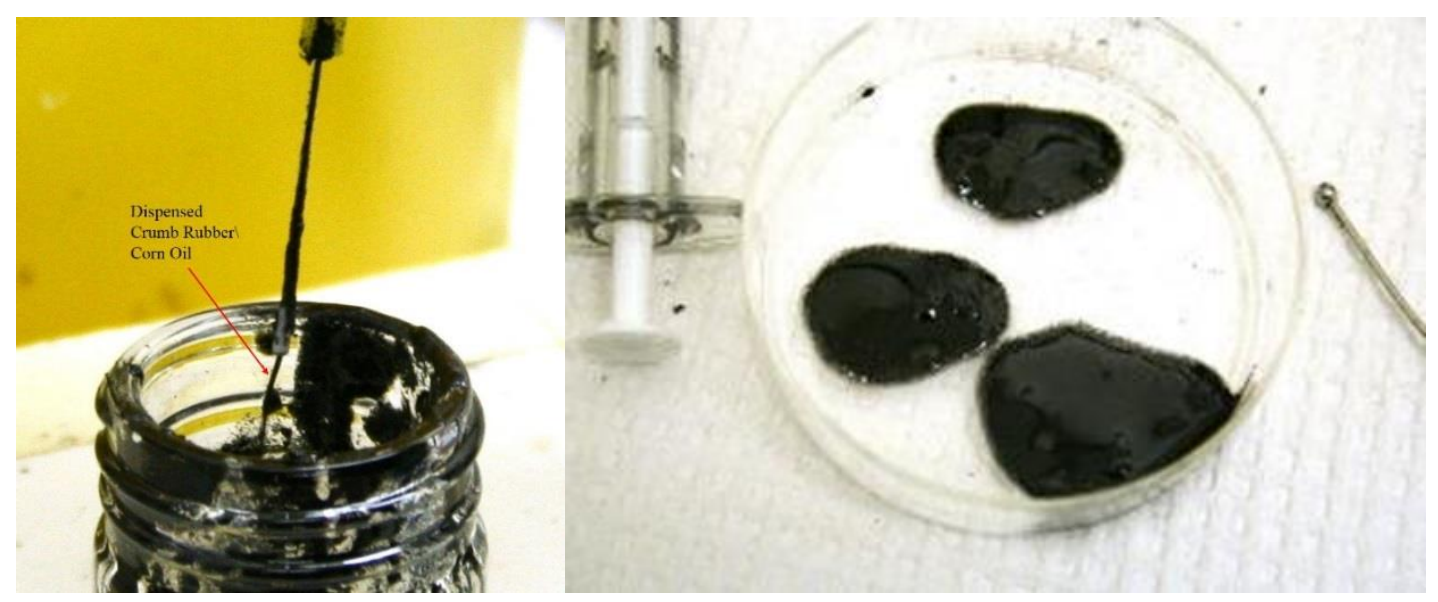

Figure 6. Representative Digital Images of 400-Mesh Crumb Rubber in Corn Oil Demonstrating Dispensing from Plastic Needle (Left) and Homogeneity (Right) 
Synthetic Turf/Recycled Tire Crumb Rubber: Feasibility Study in Support of Non-inhalation In Vivo Exposures of Synthetic Turf/Recycled Tire Crumb Rubber

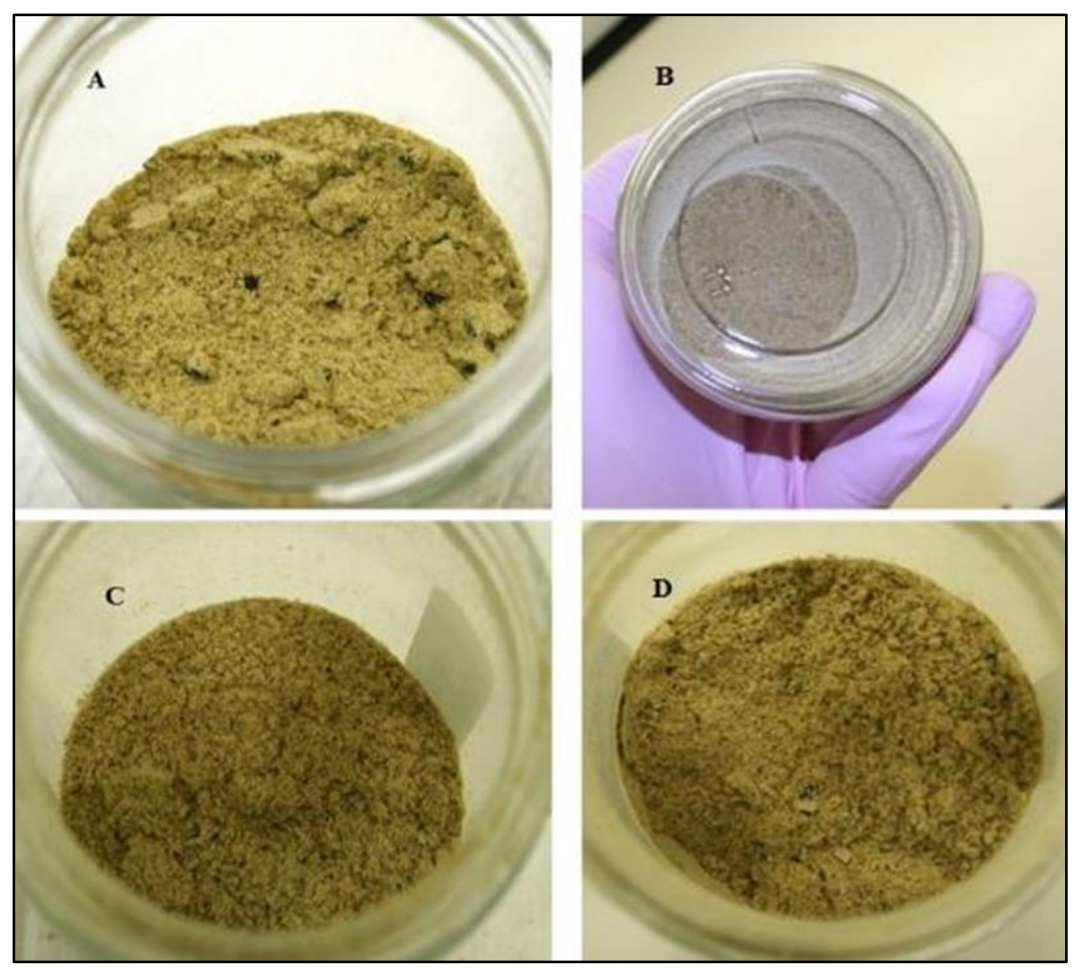

Figure 7. Representative Digital Images of Homogeneity of 14-Mesh Crumb Rubber in Feed Formulation after Shaking on Days 0 (A), 1 (B), 4 (C), and 7 (D)

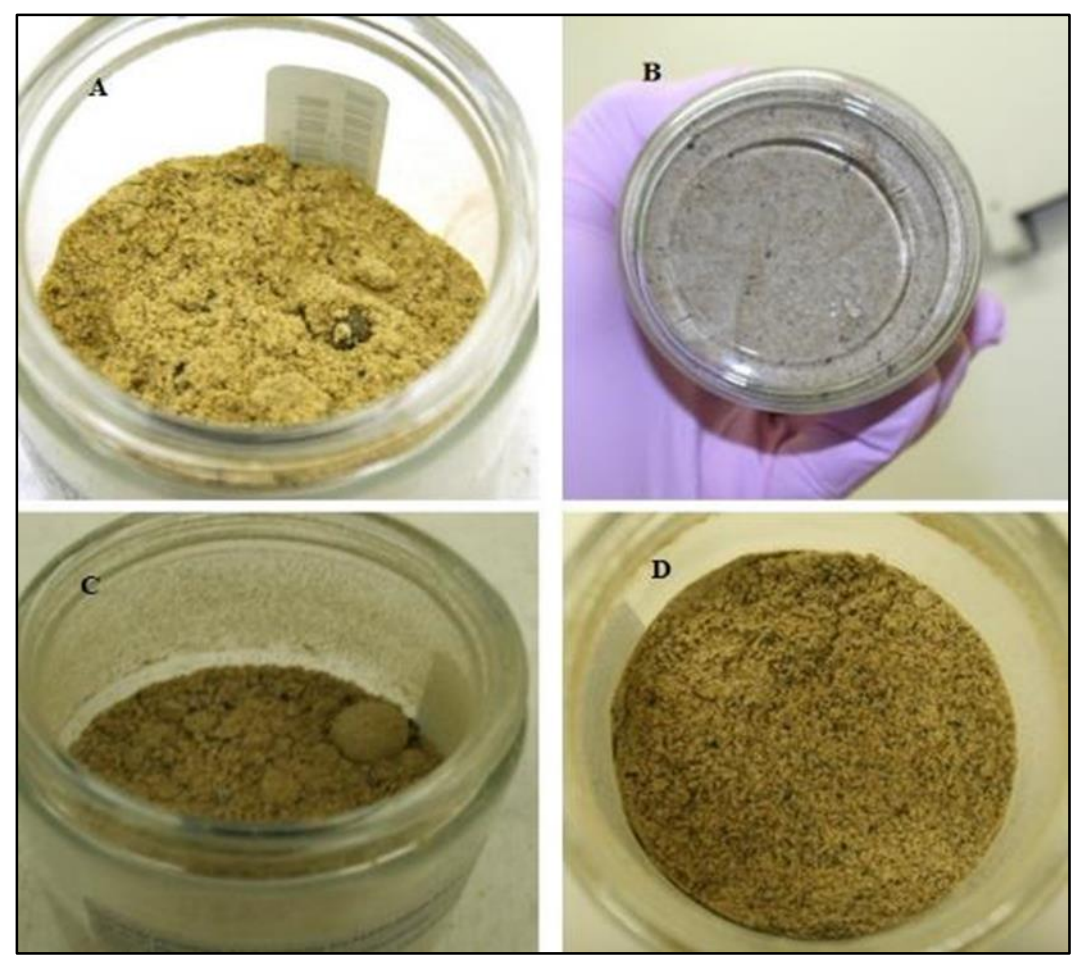

Figure 8. Representative Digital Images of the Homogeneity of the 40-Mesh Crumb Rubber in Feed Formulation after Shaking on Days 0 (A), 1 (B), 4 (C), and 7 (D) 
Synthetic Turf/Recycled Tire Crumb Rubber: Feasibility Study in Support of Non-inhalation In Vivo Exposures of Synthetic Turf/Recycled Tire Crumb Rubber

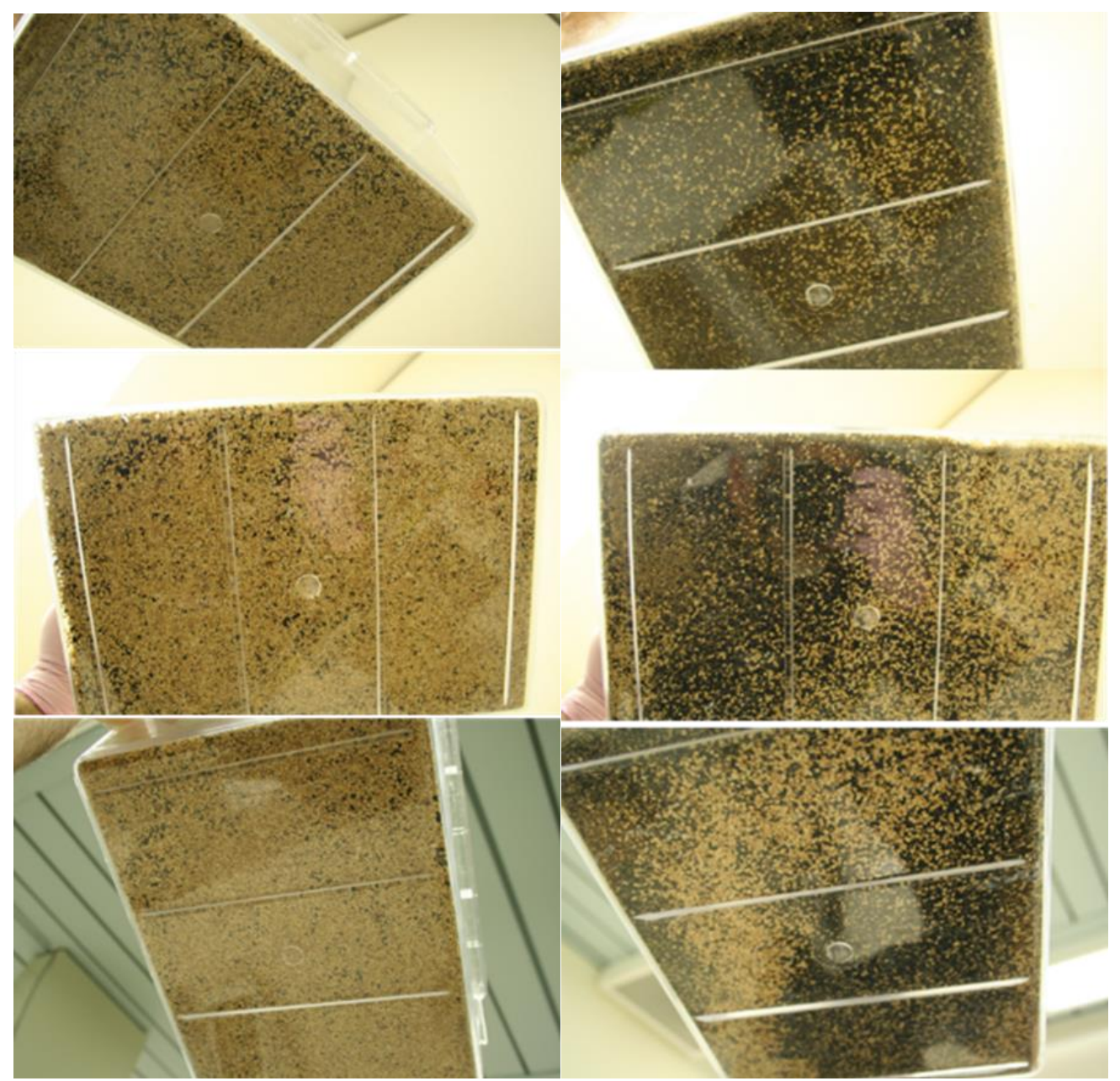

Figure 9. Representative Digital Images of Homogeneity of 14-Mesh (Left) and 40-Mesh (Right) Crumb Rubber in Bedding Formulation after Shaking on Days 0 (Top), 1 (Middle), and 4 (Bottom) 
Synthetic Turf/Recycled Tire Crumb Rubber: Feasibility Study in Support of Non-inhalation In Vivo Exposures of Synthetic Turf/Recycled Tire Crumb Rubber

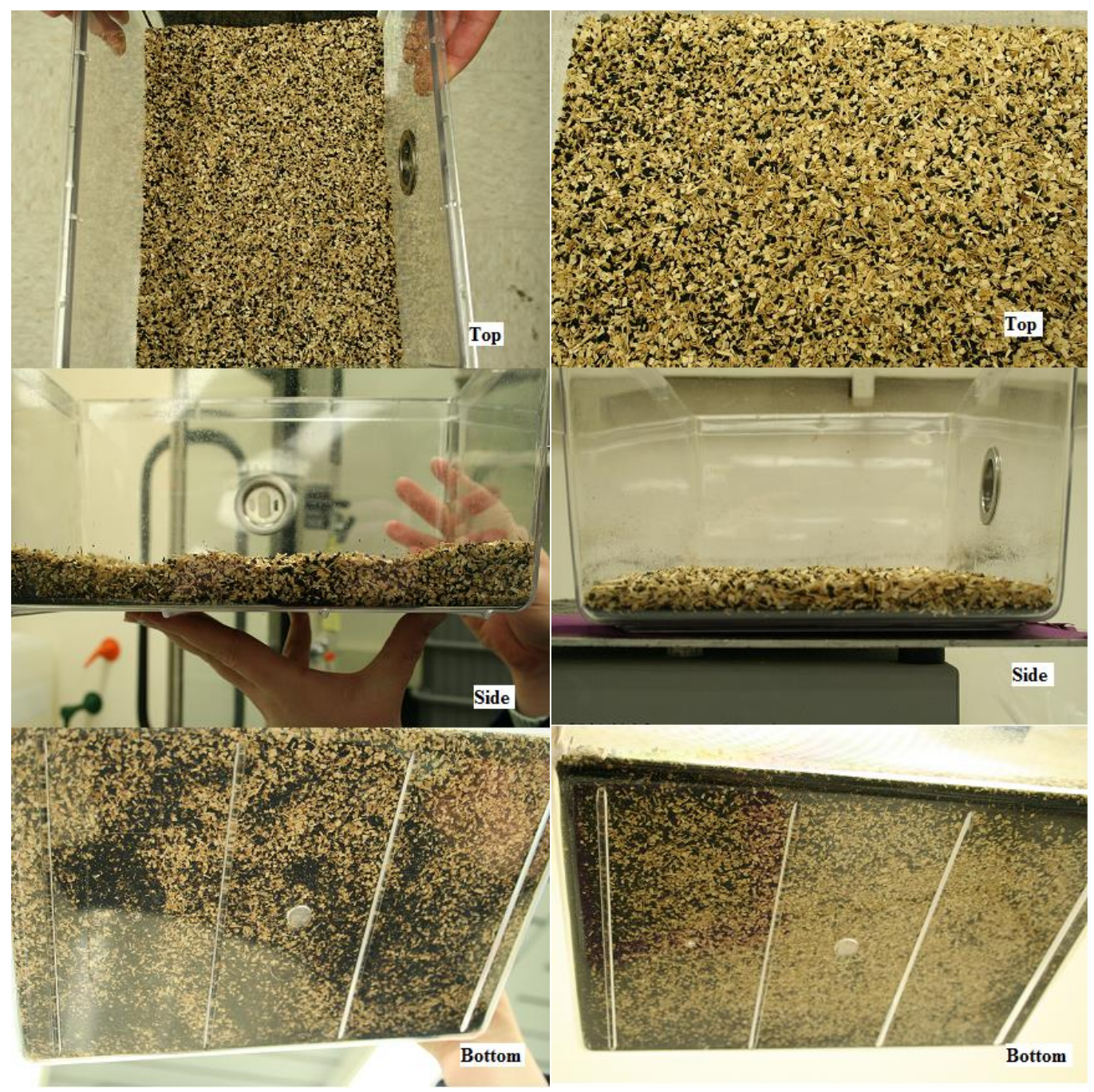

Figure 10. Representative Digital Images of Homogeneity of Combined Crumb Rubber in Bedding Formulation Before Shaking (Left) and After Shaking (Right) 
Synthetic Turf/Recycled Tire Crumb Rubber: Feasibility Study in Support of Non-inhalation In Vivo Exposures of Synthetic Turf/Recycled Tire Crumb Rubber

\section{Digital Image Recording Background Sheet}

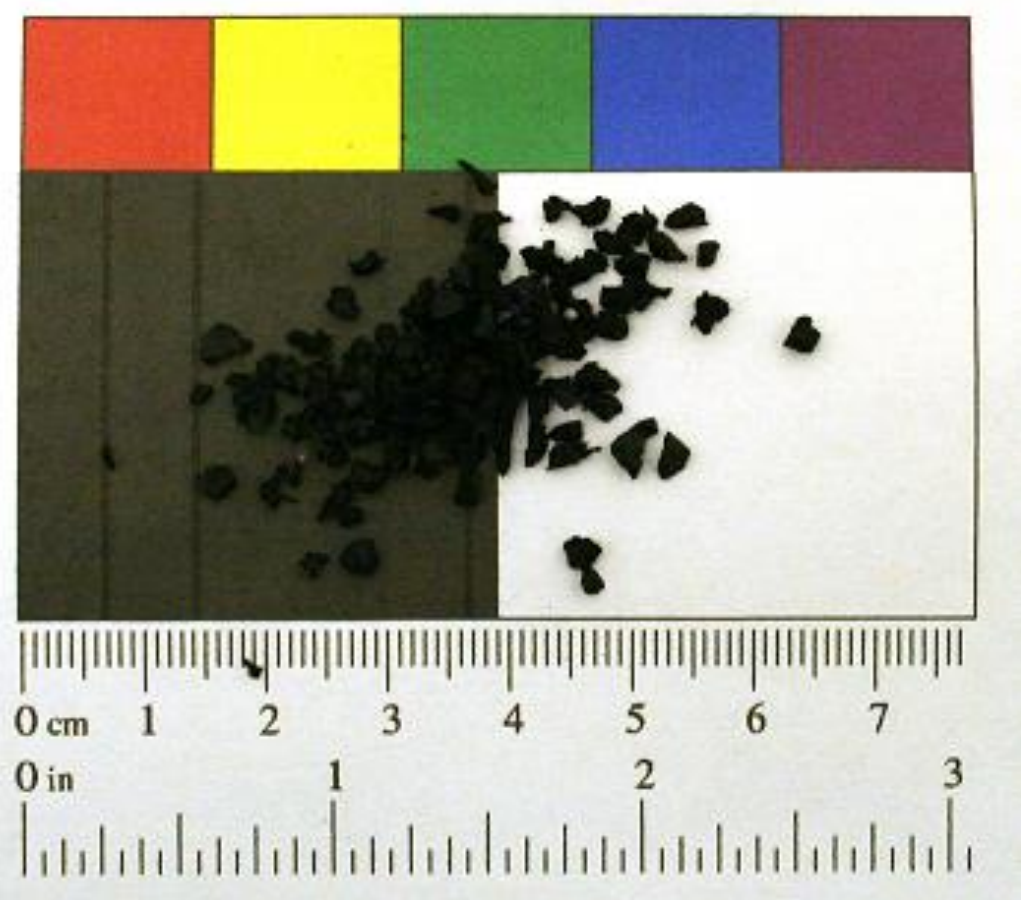

Figure 11. Digital Image of Combined Crumb Rubber Fraction

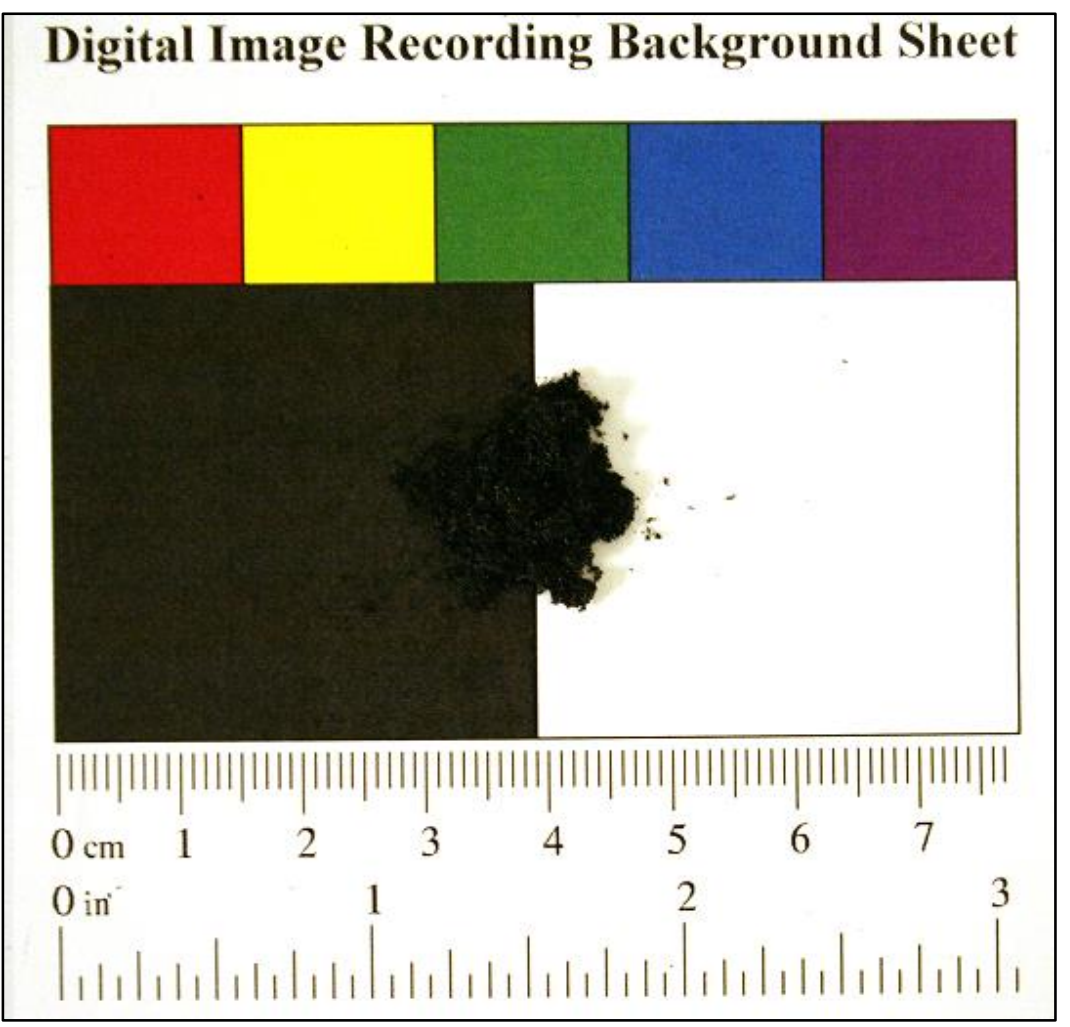

Figure 12. Digital Image of the 400-Mesh Crumb Rubber Fraction 


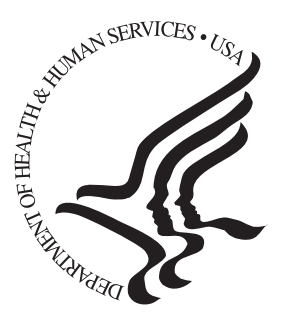

\section{National Toxicology Program}

NTP Central Data Managment, MD K2-05

National Insitute of Enviromental Health Sciences

P.O. Box 12233

Research Triangle Park, NC 27709

http://ntp.niehs.nih.gov 